\title{
Stochastic resonance in the thermohaline circulation
}

\author{
P. Vélez-Belchí ${ }^{1}$, A. Alvarez ${ }^{2}$, P. Colet, J. Tintoré \\ Instituto Mediterráneo de Estudios Avanzados (CSIC-UIB), Palma de Mallorca, SPAIN
}

R. L. Haney

Naval Postgraduate School, Monterey, USA

\begin{abstract}
A wide variety of climate records have revealed the existence of sudden a recurrent climatic changes. An important part of this variability might be related to transitions between stable equilibrium states of the thermohaline circulation. Here, we employ a box model of the ocean thermohaline circulation to show that in the presence of environmental fluctuations, a subthreshold periodic perturbation in the fresh water fluxes can induce quasiperiodic transitions between the stable states of the thermohaline circulation. This enhanced response occurs for a wide range of frequencies, including the Milankovic orbital forcing, and amplitudes. The mechanism that allows such response of the system under small perturbations arise from a nonlinear cooperation between the periodic perturbations and the fluctuations. Through this nonlinear mechanism, called stochastic resonance, significant climatic variability may be originated due to small perturbations enhanced by environmental noise and dynamics.
\end{abstract}

\section{Introduction}

The thermohaline circulation (hereinafter, THC) is that part of the global ocean circulation driven by large scale density differences. These density differences are ultimately governed by heat and freshwater fluxes at the ocean surface.The present-day THC [Gordon, 1986] is characterized by deep water formation in the North Atlantic. After formation, the deep water moves southward, at a depth of 2-3 $\mathrm{km}$, across the equator and joins the circumpolar current on its path to the Indian and Pacific Ocean, where it upwells and returns to the North Atlantic. This circulation pattern has associated a relevant transport of heat. On annual average, warm near-surface water crosses the equator northward in the Atlantic, causing a cross-equatorial volume transport of about $17 \mathrm{~Sv}$, with an associated heat transport between $0.5 \times 10^{15} W$ and $1.5 \times 10^{15} W$ [Roemmich and Wunsch, 1985]. This heat transport, largely determines important aspects of the Earth's climate [Bond, 1995; Dansgaard et al., 1993]. The important role played by the THC regulating the climate has allowed to establish a link between past climate changes and the dynamical behaviour of the THC. Specifically, recent experimental studies [Ditlevsen, 1999]

\footnotetext{
${ }^{1}$ Now at: Instituo Español de Oceanografía, Centro Oceanográfico de Canarias, Santa Cruz de Tenerife, Spain

${ }^{2}$ Now at: NATO Saclant Undersea Research Centre, La Spezia, Italy

Copyright 2001 by the American Geophysical Union.

Paper number 2000GL012091.

0094-8276/01/2000GL012091\$05.00
}

have shown, using calcium signal from the 110 kyrs GRIP (Greenland ice core project) ice-core as climate proxy, that the climate, and therefore the THC, can be represented by a double-well potential dynamical system, with the two wells representing the interstadial state and the full glacial state. Numerical models have also confirmed this multiple equilibria property of the THC [Stommel, 1961; Bryan, 1986; Marotzke and Willebrand, 1991; Thual and McWilliams, 1992; Cessi, 1994; Hughes and Weaver, 1994; Rahmstorf, 1995]. Besides the intrinsic nonlinear nature of the THC, climate records show that the THC has been also subjected to the effects of external environmental fluctuations [Bond, 1995; Ditlevsen, 1999]. In summary, the THC can be seen as a nonlinear bistable system embedded in a fluctuating environment.

Nonlinear systems characterised by a bistable nature show a rich physical behaviour in the presence of noise. One of the characteristic phenomena related to these kind of systems is called stochastic resonance (hereinafter SR). The basic idea underlying SR is that the fluctuating induced transitions between the stable states of the nonlinear system can be synchronised by very weak periodic forcings, yielding a strong enhancement of the system response [Benzi et al., 1981; Benzi et al., 1982]. Specifically, bistable systems can be described by a potential with two stable states, separated by a potential barrier. In the absence of any external forcing, the system will remain in one of the two stable states. If the bistable system is forced by noise, transitions between the two equilibrium states will occur on a mean escape time $T_{M}$ [Gardiner, 1985]. On the other hand, if the bistable system is forced by a periodic forcing, the system response will depend on the amplitude and frequency of the forcing. If the periodic forcing is too weak to cause the system to scale the potential barrier, it will remain oscillating in the neighbouring of the initial stable state. Such weak forcing is denominated sub-threshold. For forcings strong enough to overcome the potential barrier that separate the two stable states, transitions will occur between the two states with the same period of the forcing, $T_{o}$. The phenomenon of SR appears when the bistable system is driven by both, an additive random noise, for simplicity white and gaussian, and a sub-threshold periodic forcing. The weak periodic forcing modulates in time the height of the potential barrier, changing the mean escape time. A tuning of the forcing period $T_{o}$ and the mean escape time $T_{M}$ can result from this process. The system will show then transitions between the two stable states with the same period $T_{o}$ of the weak forcing and in phase with it [reviews on SR are given in Wiesenfeld and Moss, 1995].

The scope of this Letter is to show that SR is a mechanism of variability, for a wide range of periods, in the dynam- 

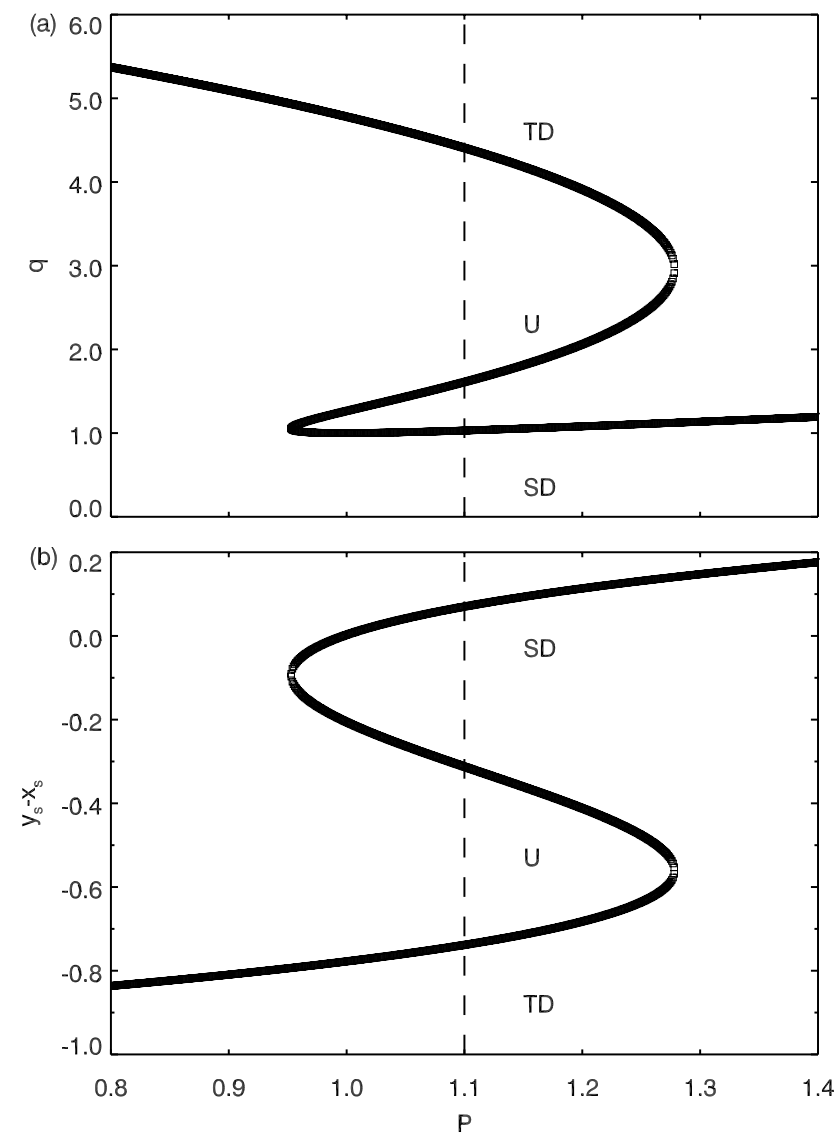

Figure 1. Steady states distribution for the nondimensional transport $q$ (a) and density difference $y_{s}-x_{s}$ (b) for the two box model using $\alpha=3.6 \times 10^{2}$ and $\mu^{2}=6.25$. The horizontal axis is the control parameter $p$, which is related to the freshwater forcing in the high latitude box (North Atlantic). The three branches found are labeled as TD (thermally driven), U (unstable) and SD (salinity driven)

ical behaviour of the THC and, in consequence, of certain aspects of climate variability.

\section{The Model and Results}

To study SR of the THC in a simple context, we have chosen the model developed by Cessi [1994]. This choice is based on previous work carried out by this author, who considered the importance of noise and found a differential stability regime, which is coherent with previous studies and observations. The model is a two box model consisting of a high latitude box and a low latitude box that models the vertically averaged circulation in a single hemisphere. Highly idealized box models have shown to be of enormous utility on understanding aspects of the long time climate variability [review is given in Ghil and Childress, 1987]. In the specific case of the THC, the box model equations can be written for the nondimensional temperature and salinity differences [Cessi, 1994] as:

$$
\begin{aligned}
& \dot{x}=-\alpha(x-1)-x\left[1+\mu^{2}(x-y)^{2}\right] \\
& \dot{y}=p-y\left[1+\mu^{2}(x-y)^{2}\right],
\end{aligned}
$$

the dot means derivative with respect to the nondimensional time $\tau=t / t_{r} ; t_{r}=220$ days is the relax- ation time scale for temperature, which is ten times bigger than the value used by Cessi [1994]. Our choice for $t_{r}$ is based on the work by Rahmstorf and Willebrand [1995]. $\alpha=t_{d} / t_{r}=3.6 \times 10^{2}\left(t_{d}=219\right.$ years is the diffusion time $)$, $\mu^{2}=t_{d} / t_{a}=6.25\left(t_{a}=35 \mathrm{yr}\right.$ is an advective time scale) and $p=\frac{\alpha_{s} S_{0} t_{d} F}{\alpha_{t} \Delta T^{*}}$ is the nondimensional freshwater forcing, where $\alpha_{t}=0.171 \mathrm{Kg} \mathrm{m}^{-3} /{ }^{\circ} \mathrm{C}, \alpha_{s}=0.781 \mathrm{Kg} \mathrm{m}^{-3} / \mathrm{psu}$, $S_{0}=35 \mathrm{psu}, \Delta T^{*}=20{ }^{\circ} \mathrm{C}$ is the difference between high and low latitude air temperature and $F$ is the freshwater flux. The nondimensional transports are calculated from the density differences, using the exchange function given by Cessi [1994].

The forcing related to the freshwater flux in (1), $p$, is considered time-dependent:

$$
p(\tau)=\bar{p}+A \sin \left(2 \pi \frac{\tau}{T}\right)+\sqrt{\varepsilon} \chi(\tau) .
$$

A value of $\bar{p}=1.1$ has been considered based on an estimate of the mean freshwater flux into the North Atlantic of about $2.3 \mathrm{~m} / \mathrm{yr}$ [Schmitt et al., 1989]. Superimposed to this mean value term, there is a periodic forcing, with amplitude $A$, and period $T$, and a Gaussian noise with zero mean and
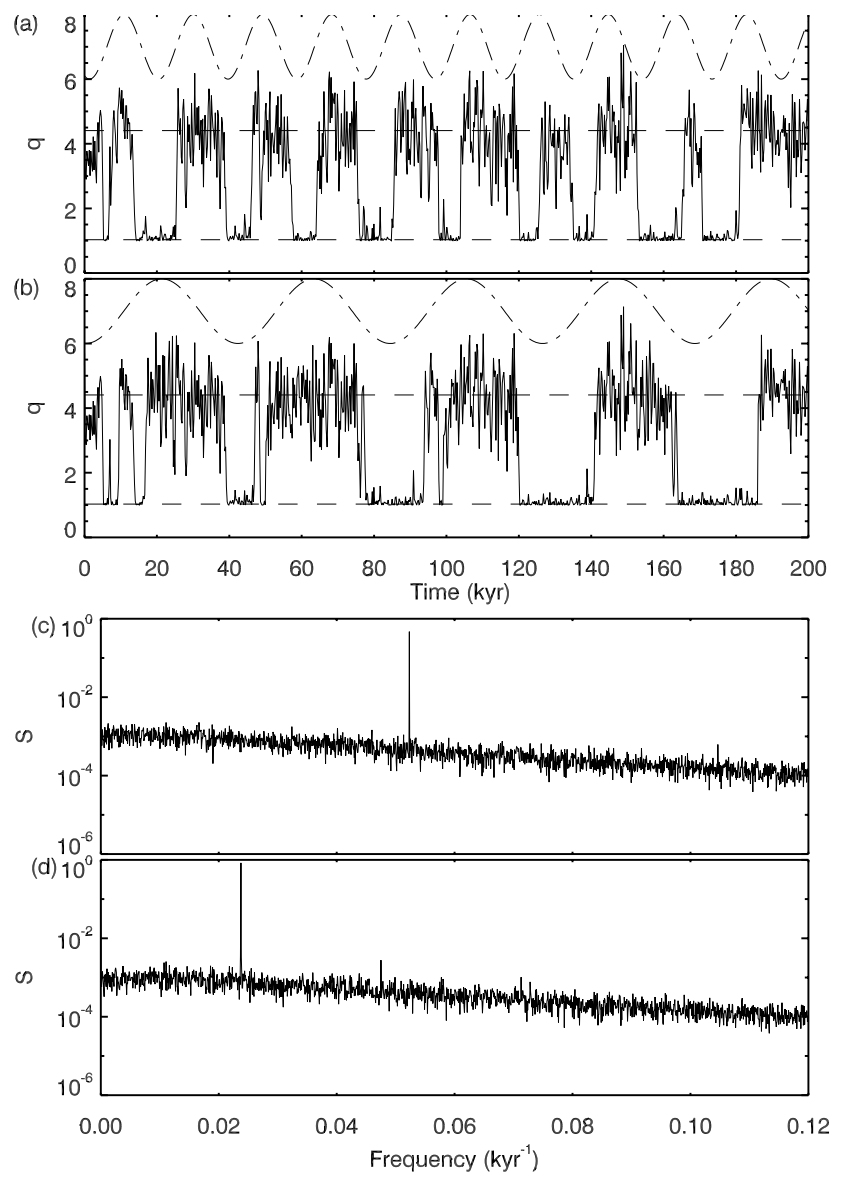

Figure 2. (a)Time series of the transport between both basins for $\epsilon=0.022\left(34 \mathrm{~m}^{2} \mathrm{yr}^{-1}\right), \mathrm{A}=0.05$ (4.45\% of the mean freshwater forcing) and $\omega=0.0727$ (corresponding to a forcing period of $\mathrm{T}=19 \mathrm{kyrs}$ ). For reference, the dashed line indicates the position of the two stable steady states, and a dot-dashed curve in the top shows the periodic forcing (note that the amplitude and mean value of the forcing has been magnified for clarity). (c) Power spectrum computed from the time series of states of (a). (b) and (d) same as (a) and (c) except for $\omega=0.0327$ ( $\mathrm{T}=42 \mathrm{kyrs}$ ). 

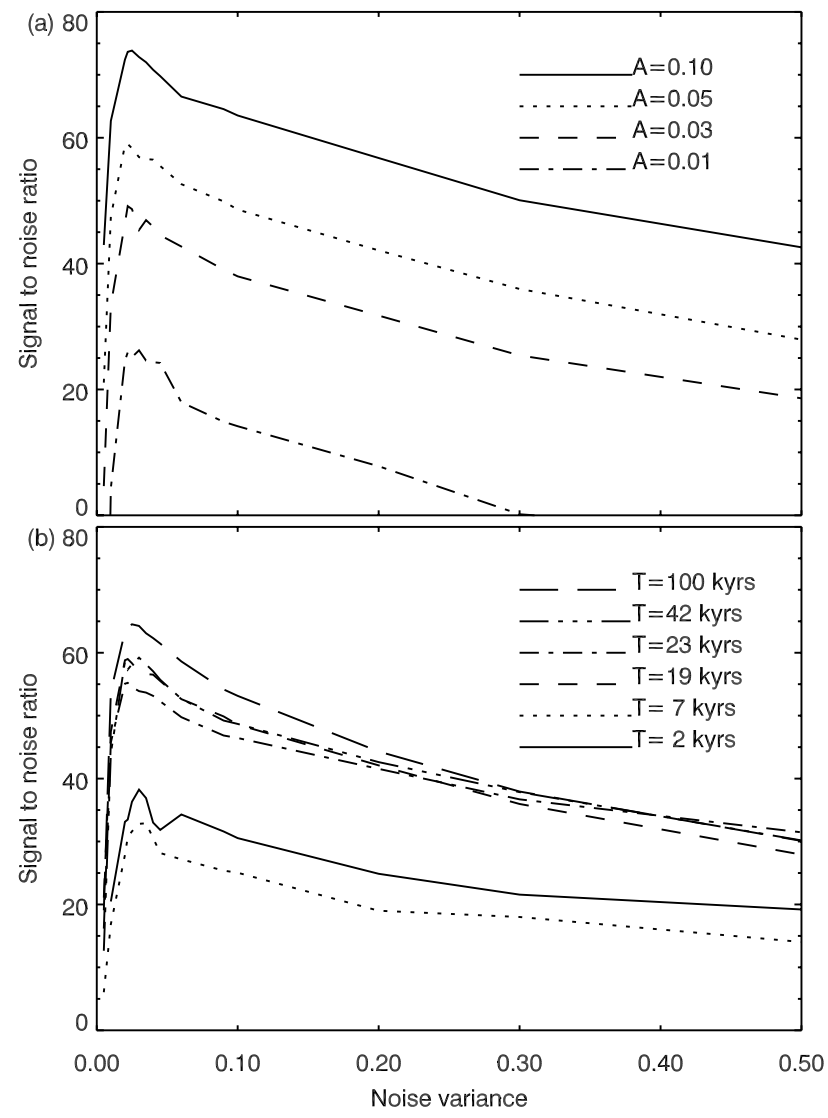

Figure 3. (a) SNR curves obtained for different amplitudes of the forcing, $\mathrm{A}=0.01,0.03,0.05,0.10$ (Corresponding to $0.90 \%$, $2.72 \%, 4.45 \%$ and $9.09 \%$ of the mean freshwater flux, respectively). The forcing frequency is $\omega=0.0727$ ( $\mathrm{T}=19 \mathrm{kyrs}$ ). (b) SNR curves obtained for $\mathrm{A}=0.05$ and different forcing periods as shown.

variance $\varepsilon$. The periodic forcing describes weak variations of fresh water fluxes with certain periodic component, like the precipitation changes over the North Atlantic induced by the Milankovic rhythms [Adams et al., 1999]. The noise term models perturbations that occur with time scales much smaller than the characteristic time scale of the system.

The model described by (1) shows a bistable behaviour with a weak and a strong transport states, representing the weak and strong thermohaline overturning respectively [Rahmstorf, 1995]. Figure 1 shows the steady solution of (1) characterized by two stable states: the thermal driven (strong transport) and the salinity driven (weak transport) THC. An unstable state is between the two stable ones. A dashline indicate the mean forcing $(\bar{p})$ and therefore the position of the two stable states for the configuration used in this study.

We have performed numerical simulations of the model (1) with the freshwater forcing given by (2). Specifically, we have used the frequencies associated with the Milankovic orbital forcing. The periods for changes in the eccentricity of the orbit, in the obliquity of the earth's axis and in the timing of the seasons relative to the earth's elliptical track nearer and further from the sun are 100 kyrs, 42 kyrs, 23 kyrs and 19 kyrs, respectively.

Figure 2a shows the dynamical evolution of the system under the combined influence of a periodic forcing with pe- riod $T=19$ kyrs, amplitude $\mathrm{A}=0.05$ (this is $4.5 \%$ of the mean freshwater flux $\bar{p}$ and four times smaller than the necessary amplitude to induce transitions in the absence of noise) and random fluctuations of variance $\varepsilon=0.022$. Jumps between the two states are clearly observed. The time series of the nondimensional transports $(q)$ were transformed into states series in the following way: for all time where $q>3$, the system is in the strong thermohaline overturning state, the state series take value 1 . While for the time where $q<3$, the system is in the weak thermohaline overturning state. The power spectrum (Fig. 2c) of the state series shows a sharp peak located at the forcing frequency, riding on a broadband noise background. This indicates that the transitions between the two stable states of the two box thermohaline circulation preferably occur with the same periodicity as the deterministic forcing. The same effect is observed for a wide range of forcing frequencies. In particular, Figures 2b, d show the results for simulations with $T=42$ kyrs, $\mathrm{A}=0.05$ (Corresponding to $4.45 \%$ of the mean freshwater flux) and random fluctuations of variance $\varepsilon=0.022$.

An appropriate way to quantify the response of the system is by measuring the signal to noise ratio (SNR) of the power spectrum at the forcing frequency, $\mathrm{S}\left(\omega_{0}\right), S N R=$ $10 \log _{10}\left(S\left(\omega_{0}\right) / B\right)$, where B is the background of the spectrum. The characteristic shape of the SNR curve is a fingerprint of the SR mechanism: SNR will increase with the noise variance, reaching a maximum value corresponding to the maximum cooperation between the signal and the noise. For large values of the noise variance the jumps will become less synchronized with the forcing and the systems behaviour will be noise dominated, showing a slow decaying SNR curve. The computed SNR curves (Fig. 3a, b) show the characteristic signature of stochastic resonance: a maximum in SNR takes place at an optimal value of the noise intensity which, for the parameters used in the present study it is around $\varepsilon=0.022$.

In Fig. 3a, b, we show the computed SNR curves for different forcing periods and amplitudes. It can be seen that SR appears for periods ranging from 2 kyrs to 100 kyrs. Focusing on the lower limit of the perturbation amplitudes, we have found SR even for perturbations less than $1 \%$ of the mean fresh water flux. This is a much smaller number than the $16 \%$ variation in the global mean precipitation, from the modern to the glacial climate, that has been found in recent simulations [Ganopolski et al., 1998]. The wide frequency range and the small amplitude required for the forcing indicates that more than an isolated phenomena, stochastic resonance in the THC could be quite common.

In summary, we have presented first evidences that a non-linear cooperative effect, SR, between the environmental noise and a weak periodic forcing in the freshwater fluxes could induce transitions between the different stable states of the THC, with a well defined time variability. Specifically, stochastic resonance has been found in band of frequencies, where the Milankovic orbital forcing is included. If true, SR could certainly contribute to the Earth's climate variability. While present results have been constrained to the study of weak periodic forcings, SR can also occur under more complicate subthreshold forcings like the chaotic ones [Collins et al., 1995]. Despite the simplicity of the model, we belive the results are robust and could be observed in more complicate and representative climate numerical models. 
Acknowledgments. We would like to acknowledge very helpful reviews of an earlier draft of this paper by Stefan Rahmstorf. This work has been funded by the Spanish CICYT (AMB95-0901) and by the Office of Naval Research, code 3220M (RLH) . P.C. acknowledge financial support from DGICYT (Spain) project PB94-1167. P.V. thanks the Spanish "Ministerio de Educación y Cultura" for a doctoral fellowship.

\section{References}

Adams J.M., M. Maslin, E. Thomas, Sudden climate transitions during the Quaternary, Progress in Physical Geography, 23, 1-36,1999.

Benzi, R., A. Sutera and A.Vulpiani, The mechanism of stochastic resonance, J. Phys. A, 14, 453-457, 1981.

Benzi, R., G. Parisi, A. Sutera and A. Vulpiani, Stochastic resonance in climatic change, Tellus, 34, 10-16, 1982.

Bond, G., Climate and the conveyor, Nature, 377, 383-384, 1995.

Bryan, F., High-latitude salinity effects and interhemispheric thermohaline circulations, Nature, 323, 301-304, 1986.

Cessi, P., A simple box model of stochastically forced thermohaline flow, J. Phys. Oceanogr., 24, 1911-1920, 1994.

Collins, J.J., C.C. Chow and T.T.Imhoff, Stochastic resonance without tuning, Nature, 376, 236-238, 1995.

Dansgaard, W., S.J. Johnsen, H. B. Clausen, D. Dahl-Jensen, N. S. Gundestrup, C. U. Hammer, C. S. Hvidberg, J. P. Steffensen, A. E. Sveinbjörnsdottir, J. Jouzel, and G. Bond, Evidence for general instability of past climate from a 250-kyr ice-record, Nature, 364, 218-220, 1993.

Ditlevsen, P. D., Observations of $\alpha$-stable noise induced millennial climate changes from ice-core record, Geophys. Res. Lett., 26, 1441-1444, 1999.

Ganopolski A., S. Rahmstorf, V. Petoukhov, M. Claussen, Simulation of modern and glacial climates with a coupled global model of intermediate complexity, Nature, 391, 351-356, 1998.

Gardiner, C. W.Handbook of stochastic methods for physics, chemistry and the natural sciences., 442pp., Springer-Verlag, 1985.
Ghil, M., and S. Childress, Topics in Geophysical Fluid Dynamics: Atmospheric Dynamics, Dynamo Theory, and Climate Dynamics., 485 pp., Springer-Verlag, 1987.

Gordon, A.L., Interocean exchange of thermocline water, J. Geophys. Res., 91, 5037-5046, 1986.

Hughes, T.M.C., and A.J. Weaver, Multiple equilibria of an asymmetric two-basin ocean model, J. Phys. Oceanogr., 24, 619637, 1994.

Marotzke, J., and J. Willebrand, Multiple equilibria of the global thermohaline circulation, J. Phys. Oceanogr., 21, 1372-1385, 1991.

Rahmstorf, S., Bifurcations of the Atlantic thermohaline circulation in response to changes in the hydrological cycle, Nature, 378, 145-149, 1995.

Rahmstorf, S., and J. Willebrand, The role of temperature feedback in stabilizing the thermohaline circulation, J. Phys. Oceanogr., 25, 787-805, 1995.

Roemmich, D.H., and C. Wunsch, Two transatlantic sections: Meridional circulation and heat flux in the subtropical North Atlantic ocean, Deep-Sea Res., 32, 619-664, 1985.

Schmitt, R. W., P. S. Bogden, and C. E. Dorman, Evaporation minus precipitation and density fluxes for the North Atlantic, J. Phys. Oceanogr., 19, 1208-1221, 1989.

Stommel, H.,Termohaline convection with two stable regimes of flow, Tellus, 13, 224-230, 1961.

Thual, O., and J.C. McWilliams, The catastrophe structure of thermohaline convection in a two-dimensional fluid model and a comparison with low-order box models, Geophys. Astrophys. Fluid Dyn., 64, 67-95, 1992.

Wiesenfeld K., and F. Moss, Stochastic resonance and the benefits of noise: from ice ages to crayfish and SQUIDs. Nature, 373, 33-36, 1995.

Pedro Joaquín Vélez Belchí, Instituo Español de Oceanografía, Centro Oceanográfico de Canarias, Crta San Andrés s/n, E-38120 Santa Cruz de Tenerife, Spain. (e-mail: pvb@ieo.rcanaria.es)

(Received July 21, 2000; accepted January 30, 2001.) 